\title{
La Educación Primaria en el Cádiz de las Cortes
}

\section{GLORIA ESPIGADO TOCINO}

Dentro de un campo de investigación más amplio, que trataría de reproducir las condiciones educativas del Cádiz ochocentista, avanzamos en las siguientes páginas la realidad escolar y el debate ideológico suscitado en nuestras primeras Cortes liberales en torno a los cambios que la nueva concepción de la educación, nacida en el primer ciclo revolucionario europeo contemporáneo, habría de adoptar nuestro país, en clara correspondencia con las tendencias de ruptura política que se ponen de manifiesto en el período bélico de la Guerra de la Independencia y que encarnarían la labor legislativa desarrollada por el parlamentarismo gaditano doceañista (1). Ofrecemos una aproximación a los ideales educativos de aquella representación nacional, analizando sus proyectos y realizaciones en este apartado y, más allá de utopías y textos legales, interesados en la reconstrucción del día a día escolar en los comienzos del siglo, trataremos de describir la escuela gaditana de aquel entonces, reconociendo cuantas había, cómo y dónde se educaban los niños y las niñas, con qué libros, con qué objeto, con qué maestros, bajo qué jurisdicción. etc. Es por ello que, si bien serán expuestos los logros que en este capítulo pueden ser adjudicados a la labor reformista de las Cortes gaditanas, no dejaremos de interrogarnos acerca de cuál fue la sensibilidad que mostraron ante dichas reformas nuestras autoridades locales, lo que incluye hacer referencia tanto a su reflexión teórica sobre la importancia que en las naciones avanzadas iba adquiriendo el concepto de instrucción universal, como a la respuesta práctica que dieron ante esos requerimientos educativos que traían los nuevos tiempos (2).

(1) Sería prolijo hacer una mínima alusión a los trabajos que se han centrado en un período histórico de preferente interés para los contemporaneístas. Los nombres que solemos relacionar con su estudio son los de Federico Suárez Verdaguer, Miguel Artola, Josep Fontana o, más recientemente; la Tesis doctoral defendida por Manuel Morán Ortí, Poder y gobierno, en las Cortes de Cádiz (1810-1813), 1986. Para más información bibliográfica Vid. José Andrés Gallego, "El proceso constituyente gaditano: cuarenta años de debate", en Gades. Extraordinario CLXXV aniversario de la Constiución de 1812, № 16, Diputación Provincial de Cádiz, 1987, pp. 119-140.

(2) Sobre las condiciones generales de la educación en la primera mitad del XIX, RUIZ BERRIO, J., Política escolar de España en el siglo XIX (1808-1833), Madrid, C.S.I.C., 1970; VIÑAO FRAGO, A, Política y educación en los orígenes de la España Contemporánea, Madrid; Siglo XXI, 1982; GÓMEZ MORENO, A., Liberalismo y educación primaria en España (1838-1857), Secretariado de Publicaciones de la Universidad de Zaragoza, 1990.

DOI: http://dx.doi.org/10.25267/Trocadero.1995.16.10 


\section{EL MARCO ESPECULATIVO}

Los trabajos preparatorios para alumbrar una futura ley de instrucción tienen sus comienzos en el nombramiento de una Junta, por parte de la Central, con fecha 2 de junio de 1809, con el encargo de presentar un proyecto ante las Cortes. Jovellanos, que la preside, redactaría sus famosas Bases para la formación de un plan de instrucción pública, como orientación preliminar para la misma. Posteriormente, solventada la redacción constitucional, se designaría una nueva Junta con el mandato de remitir a la Comisión de Instrucción Pública de las Cortes un plan general de instrucción, a cuyo amparo nacería el famoso Informe para proponer los medios de proceder al arreglo de los diversos ramos de instrucción pública atribuido al poeta y tribuno doceañista Manuel José Quintana y rubricado en Cádiz el 9 de septiembre de 1813. Una vez en manos de la Comisión, ésta establecería el Dictamen sobre el proyecto de Decreto de arreglo general de la Enseñanza pública el 7 de marzo de 1814, ya sin tiempo para su discusión parlamentaria, por cuanto que el regreso de Fernando VII truncaría la labor legislativa de las Cortes (3).

Comenzando, como debe hacerse, por el principio, será preciso señalar que no podemos considerar como original las ideas contenidas en el texto constitucional o en el propio Informe del diputado Quintana ya mencionado. Nuestro primer liberalismo lo único que hace, como lo hizo en su día la Asamblea revolucionaria francesa, fue escuchar con atención lo que, al respecto, ya decían los ilustrados y asumir la proyección sobre la sociedad de sus máximas de universalización, uniformización y estatalización de la enseñanza, cuando menos en sus niveles primarios. No en vano, los expertos coinciden en señalar que este texto o, anteriormente, los famosos escritos del asturiano Melchor Gaspar de Jovellanos, se inspiran directamente en el Las cinco memorias sobre instrucción pública (1791) o en el Rapport et projet de Decret sur l'organisation générale de l'instruction publique (1792) de Condorcet, cuando no, todos tenemos en mente el proyecto utópico de educación perfecta ideada por Rousseau en su Emilio publicado hacia 1762 (4).

La Ilustración, por tanto, como movimiento intelectual, no sólo se detuvo en resaltar la importancia de la extensión de la instrucción, más o menos elemental, a todo el mundo, sino que, como proposición política reconoció como imprescindible para la felicidad del Estado la educación de la ciudadanía. No olvidemos

(3) DELGADO CRIADO, B.(coord.), Historia de la educación en España y América. La Educación en la España Contemporánea (789-1975). Madrid, Ediciones SM, 1994, pp. 41-49. Tanto el texto de las Bases redactadas por Jovellanos, así como el Informe de Manuel José Quintana se encuentran compilados en Historia de la Educación en España. Del despotismo ilustrado a las Cortes de Cádiz, Vol.I, Madrid, Ministerio de Educación y Ciencia, 1979, pp. 351-374 y $377-419$, respectivamente. El Dictamen de la Comisión puede verse en la misma colección, De las Cortes de Cádiz a la revolución de 1868, Madrid, Ministerio de Educación y Ciencia, Vol.II, 1979, pp. 353-376.

(4) De enorme interés para rastrear los débitos de nuestro primer proyecto liberal, OSSEMBACH SANTER, G. y PUELLES BENITTEZ, M., ed., La Revolución Francesa y su influencia en la educación en España, U.N.E.D. y Universidad Complutense de Madrid, 1991. 
que en nuestro propio proyecto constitucional (Art. 25) se preveía la pérdida de los derechos políticos adquiridos a todos aquellos que no supieran leer y escribir a partir de 1830. Educar para qué, pues sencillamente, no nos engañemos, no para subvertir el orden social establecido sino para preservarlo de una forma inteligente. Hacer progresar a una sociedad donde cada uno ha comprendido el deber de realizar la misión que tiene encomendada con el grado mayor de eficacia posible. Para que cada ciudadano conozca los derechos que le asisten, así como las obligaciones a las que se debe, de forma que perturbaciones no deseadas puedan evitarse. Así pues, si en nuestro primer constitucionalismo lo que prima es la idea del ciudadano convenientemente educado para el ejercicio de sus obligaciones, en etapas posteriores, cuando la soberanía nacional torne popular y se abra paso el sufragio universal masculino la necesidad expresada será la de capacitar al votante para la distinción de la opción correcta ante las urnas, neutralizando el peligro que siempre se da por sentado que existe entre la ignorancia y la revolución social. Mujeres instruidas también, no tanto para que participen de las ventajas jurídicas que introduce esa sociedad de ciudadanos y no de privilegiados, sino para que estén en condiciones de transmitir a sus hijos este cúmulo de principios que garantizarán la predisposición posterior al buen servicio a la patria, al Estado y a su Majestad (5).

Por tanto, universalizar los conocimientos básicos, en lo que más adelante se va a entender como educación elemental y ahora se sigue denominando estudios de primeras letras, a todo el conjunto social, aunque preservando los estudios superiores, universitarios, a una elite, a una minoría rectora, elegida en función de la cuna o del talento demostrado, según se contemple un mayor o menor grado de igualdad de oportunidades para poder escalar posiciones, y crear un compartimento de instrucción intermedia, donde prime el carácter práctico de las materias impartidas, para una clase media necesitada de aplicar unos conocimientos menos literarios y más científicos que los que ofertaban, por entonces, los estudios de humanidades o de latinidad, nuestros antecedentes inmediatos de esa segunda enseñanza que se instalará en los institutos diseñados por el Estado ochocentista burgués con esta finalidad. Tres etapas como vemos, tres niveles de formación que, en su enunciado, al menos, han llegado hasta nosotros y que pueden presentar vasos comunicantes entre sí, facilitando u obstaculizando la promoción social (6).

(5) Se nos ha explicado que el Informe Quintana excluye a las mujeres de sus disposiciones, circunscribiéndolas a lá educación, que no instrucción, que pudiera proporcionarle el espacio privado y doméstico, de forma que habrá que esperar a la Ley Moyano de 1857, para encontrarnos con un texto legal que estipule las condiciones, ya públicas, de su educación. BALIARÍN, Pilar, "La construcción de un modelo educativo de "utilidad doméstica", en DUBY, G. Y PERROT, M., Historia de las mujeres IV, Madrid, Taunus, 1992, p.600. Por su parte, el Dictamen de la Comisión parlamentaria, vendría a ratificar la opinión anterior, reconociendo la importancia de educar al ser que "apoderado casi exclusivamente de la educación del hombre en su niñez, tiene un gran influjo en la formación de sus primeros hábitos", por lo que convenía al Estado costear algunos establecimientos donde se enseñara a las niñas a leer, a esçribir y "las labores propias de su sexo", Historia de la Educación en España... Op. Cit, Vol. II, p.373.

(6) En el Dictamen se prescribía una primera educación "universal", para todos, una segunda "bastante general" y una tercera que no será ya ni universal, ni general, sino "particular y reducida"... Ibidem, pp. 364 . 
Hemos dicho universalización pero también uniformización de los conocimientos. Hay que atenerse a unos sistemas, a unos textos y a unas materias comunes para cada nivel especificado, para cada titulación que se pretende alcanzar. El objetivo es la felicidad de un pueblo obedeciendo a su soberano y el camino hasta llegar a dicha meta no admite bifurcación posible. Se abre paso decididamente en el nacimiento del Estado burgués la idea de que se gobierna mejor lo que es homogéneo, se pretende terminar con las disparidades de foros, costumbres, tradiciones, etc. La opción centralizada del poder se hace bien patente y su primera demostración será el acatamiento para todos de un texto fundamental, una mismas fiestas y celebraciones que identifiquen al nuevo poder, más adelante, unas mismas pesas y medidas, pero también, y sobre todo, para los primeros estadios de instrucción general, unos mismos métodos, unos mismos textos y unos saberes iguales (leer, escribir y contar, como proponía Quintana) y, evidentemente, por encima de la pluralidad de decretos y ordenanzas reales, el proyecto de Quintana es el primer paso para reglamentar los diferentes niveles educativos en un único compendio legal.

Se abre paso también un nuevo sentido de lo público aplicado a la educación y que responde a la decidida convicción, aunque se podrían incluir matices al respecto, de estos ilustrados liberales, expresión que recabaría las fuentes dieciochescas en las que beben, de adjudicar la responsabilidad rectora en materia educativa al nuevo Estado naciente, lo que evidentemente conduce, a poco que conozcamos quién posee hasta el momento los canales de instrucción del país, a un enfrentamiento con la Iglesia como institución convencida del derecho que le asiste para proporcionar formación cristiana y religiosa junto a los saberes literarios de lectura y escritura al conjunto de los españoles (7).

No deberíamos interpretar que se produce una lucha abierta entre estos dos sectores, seglar y religioso, con pretensiones diferentes. Todos o casi todos los autores coinciden en destacar el respeto, frente a otros modelos evolutivos más radicales como el francés, de nuestro primer liberalismo para con las instituciones eclesiásticas, recogidas en la confesionalidad que expresa la Constitución de 1812 y, desde luego, en lo que aquí nos atañe, en el reconocimiento, no tácito, ni implícito, sino claramente formulado de que una educación, que no sólo instrucción, quedaría incompleta sin que los principios morales del cristianismo penetren en las conciencias de los hombres desde su más tierna edad. Sin embargo, lo que sí, desde luego, se va formulando es la declaración más contundente de quién tiene la jurisdicción educativa, que ya es concebida como un asunto temporal, que implica el protagonismo de las instancias del poder público. No obstante, aunque esta idea se expresa por primera vez en proyecto legal, no pode-

(7) PUELLES BENÍTEZ, M., de, Educación e ideología en la España contemporánea (1767-1975), Barcelona, Labor, 1980 , p.53, es partidario de marcar las diferencias entre el proyecto ilustrado y el liberal, encontrando al primero más circunscrito a las transformaciones económicas y al segundo más decidido en las implicaciones políticas que sugiere el parlamentarismo del nuevo régimen. Sin embargo, aun aceptando estas pertinentes puntualizaciones, no podemos obviar el paralelismo entre los dos discursos, defendidos por personalidades culturalmente formadas en el siglo de las luces, que asisten y, aún más, ayudan a generar en la coyuntura doceañista los cauces institucionales para afrontar la reforma. 
mos llegar a pensar que este nuevo concepto de publicidad es igualmente entendido por unos y por otros. La confusión en torno a la expresión "educación pública" en los textos de variada naturaleza que hemos manejado es evidente y predomina una acepción más tradicional, más propia del pasado, aquella que entiende por enseñanza pública la que se imparte no en la privacidad del hogar, del ayo que instruye al alumno en propio domicilio, sino aquella que se oferta, bien por organismos del Estado, bien por particulares, eclesiásticos o no, al pueblo en igeneral. Como el propio Informe presentado a las Cortes decía "que la enseñanza sea pública, esto es, que no se dé a puertas cerradas, ni se limite sólo a los alumnos que se alistan para instruirse y ganar curso", en definitiva, que sea reglada en establecimientos abiertos al exterior. Por tanto, las ideas que hoy manejamos acerca de la enseñanza privada, como aquella que es impartida por particulares y enseñanza pública, aquella que asume el Estado, estaba en sus primeros estadios de definición y ni siquiera el legislador parecía sensible a esta distinción.

Universalización, uniformización, estatalización y gratuidad, para, al menos, el primer nivel necesariamente común pero no necesariamente obligatorio, puesto que habrá que esperar hasta la Ley de Claudio Moyano de 1857 para que se establezca la obligación de acudir a la escuela entre los 6 y los 9 años. Antes de que nos llegue esta normativa compulsiva, el poder público, consciente también de sus problemas financieros para hacer factible el acceso gratuito de la enseñanza elemental a toda la ciudadanía, se contentará con recomendar y alentar la apertura de centros por todo el país que ejerciten esa acción benéfica de ofrecer los saberes esenciales: sociedades económicas, establecimientos religiosos, etc., por lo que el deseo de facilitar sin coste alguno la instrucción correspondiente a los primeros niveles, que tanto el Informe como el Dictamen de la Comisión persiguen, se estrellará contra la falta de recursos crónica de la Hacienda, no resuelta, tampoco, en la Ley de 1857 , por cuanto que la obligatoria escolarización de determinado segmento de edad entra en contradicción con el reconocimiento de las retribuciones que pueden solicitar los maestros de los alumnos que asisten a la escuela pública (8).

Vistas las innovaciones más representativas que en materia de educación nos aporta nuestra primera etapa constitucional, será el momento oportuno para hacer el repaso cronológico de cómo y en qué momento se presentan a la luz pública dichas reformas. Ya hemos adelantado que pese a que la preocupación por la formación de los ciudadanos está presente en los ensayos y reflexiones de nuestros ihustrados, como Feijoo, Campomanes, Cabarrús, Jovellanos o el propio Quintana, es cierto que la materialización en un corpus legal de todas estas inquietudes se resume, en todo el periodo de invasión francesa y de legislación gaditana, en varios escritos claves, alguno de los cuales ya han sido citados.

(8) Ley de Instrucción Püblica de 9 de septiembre de 1857, en Colección legislativa de España, Imprenta del Ministerio de Gracia y Justicia, Madrid, 1857. En el Título I, Art. 9 se decía que sería gratuita sólo para los padres que no pudieran costearla (p.267), pero en la Sección $3^{2}$, Capítulo I, Art. 192, relativa al magisterio, disponía que éste, además del sueldo fijo, tenía derecho a sumar el producto de las "retribuciones" que serían fijadas por la autoridad competente y que provendrían de los niños pudientes que acudiesen a su clase (p.298). 
Comenzando por el dictado de nuestra primera Constitución habrá que referirse a lo expresado en el título IX, artículos 366 a 371, en los que se recoge el deseo de universalizar los conocimientos elementales (Art. 366), primando la homogeneidad de contenidos y métodos, que incluye la obligatoria explicación de un catecismo civil (Art. 368), centralizando y atribuyendo la labor de control a un cuerpo directamente subordinado a las directrices del gobierno (Art. 369) y estableciendo la jurisdicción pública en materia de legislación educativa al hacerla depender de las decisiones dirimidas en el seno parlamentario (Art. 379). Por último, curiosamente relacionado con la reglamentación fundamental adjudicada a la enseñanza, el artículo 371 consagraba el derecho a la libertad de expresión (9).

Otro documento imprescindible para el estudio de los primeros reglamentos liberales aplicados a la instrucción es el mencionado Informe Quintana, como hemos dicho muy sujeto a los ideales de Jovellanos expresados en sus Bases y sobre todo a los que el girondino Condorcet en su Rapport hiciera público ante la Asamblea revolucionaria francesa, allá por 1792. Sorprende que el Informe del diputado Quintana sea encargado y presentado a las Cortes legislativas en una fecha tan tardía que se remonta a los últimos meses de 1813, posteriormente, el final de la guerra, la expulsión del invasor y la llegada del "deseado", con la involución política que de todos es conocida, impediría su discusión parlamentaria quedando anulada cualquier posibilidad de puesta en vigor. Concretamente este Informe emitido por la Junta que creara en marzo de 1813 la Regencia para "proponer los medios de proceder el arreglo de los diversos ramos de instrucción pública", será, después de elaborado, remitido a las Cortes para su discusión y debate en septiembre de 1813 e, iniciada la segunda legislatura, a finales de febrero de 1814 se esperaba la discusión del Dictamen a que dio lugar cuando el retorno al absolutismo truncó el inicio de esta reforma educativa y del proyecto liberal en general. En puridad se trataba de un compendio de todos los principios que ya aquí hemos enumerado: principio de universalidad, uniformidad, publicidad (sin olvidar el matiz que hemos introducido algo más arriba), gratuidad y libertad, al señalar que cada uno estaría en disposición de adquirir los conocimientos dónde y con quién le pareciera oportuno, al reconocer la imposibilidad para el Estado de ofertar la totalidad de las plazas demandadas. Esbozada quedaba, también, la libertad para la libre concurrencia entre los maestros, en donde, más que una apuesta por la libertad de cátedra, primaba el sentido de suprimir viejas restricciones gremiales dentro de la profesión.

(9) El articulado referido se expresaba del modo siguiente:

Art. 366.- En todos los pueblos de la Monarquía se establecerán escuelas de primeras letras, en las que se enseñarâ a los niños a leer, escribir y contar, y el catecismo de la religión católica, que comprenderá también una breve exposición de las obligaciones civiles.

Art. 368.- El plan general de enseñanza será uniforme en todo el reino, debiendo explicarse la Constitución política de la Monarquía en todas las Universidades y establecimientos literarios, donde se enseñen las ciencias eclesiásticas y políticas.

Art. 369.- Habrá una Dirección General de estudios, compuesta de personas de conocida instrucción, a cuyo cargo estará, bajo la autoridad del gobierno, la inspección de la enseñanza pública.

Art. 370.- Las Cortes por medio de planes y estatutos especiales arreglarán cuanto pertenezca al importante objeto de instrucción pública. 
El proyecto doceañista estaría abocado a esperar mejores tiempos y será en el Trienio Constitucional, con la recuperación por parte de los liberales de las instancias del poder, cuando con ligeras variaciones se transforme en norma legal en 1821. Antes de esta fecha, la reacción absolutista fernandina nos volvería a sumir, con el decreto de 4 de marzo de 1814 que anulaba la Constitución y la obra legislativa de las Cortes, 'en el viejo plan de 1771 que mantenía las formas propias del Antiguo Régimen en materia educativa. Aún más, dando un paso atrás en la gestión pública de la docencia, con fecha de 19 de noviembre de 1815, alegando dificultades en el tesoro real, se disponía que la Iglesia podía y debía en todas sus fundaciones piadosas suplir la insuficiencia de los poderes temporales haciéndose cargo de la enseñanza primaria, sobre todo (10).

Suficientemente expuesto el panorama ideológico y teórico que rodea la coyuntura histórica de la Guerra de la Independencia y del primer liberalismo, es hora que pasemos a apreciar, en primer lugar, la permeabilidad que mostraron nuestras autoridades locales, tan cercanas al teatro de la discusión asamblearia, y, por otro, puesto que la experiencia nos enseña que los textos legales, los proyectos y las reformas suelen ir bastante por delante de lo que la realidad cotidiana se permite cambiar, habrá que comparar dichos proyectos con el panorama educativo atribuible a nuestra ciudad (11).

El Cabildo municipal, por su parte, tendrá también la oportunidad de pronunciarse con respécto a las bases que considera deben formar parte del proyecto educativo que las Cortes tienen que elaborar. Su informe, que precede al que Manuel José Quintana presentará en 1813, tiene fecha de 1809 y el enorme interés de adelantar muchas de las propuestas que más adelante el insigne poeta $\mathrm{y}$ tribuno incluirá en su trabajo. De nuevo, nos encontramos ante un texto ilustrado, en algunos aspectos con algunas innovaciones respecto al que será proyecto nacional (12). La universalización y uniformización se dan por seguras, así como la gratuidad de los primeros estadios que deben estar garantizados por los poderes públicos. Otra cosa va a ser la consideración que tenga la enseñanza superior a la que se reconoce pareja, y cito textualmente "a las conveniencias que el nacimiento o la fortuna les ha proporcionado" muy en la línea de lo que es el pensamiento de la época, por lo demás (Vid. nota 6 del presente trabajo). Aparte de esto, resulta enormemente interesante que por fin nos encontremos con un documento que además de preocuparse por el grado de aptitud del futuro maestro y su idoneidad a la hora de obtener el título correspondiente, se ocupe, de forma novedosa, de idear un sistema para formarlo en sus funciones, proponiendo en concreto la creación de cátedras de educación en las universidades, en una versión que podría acercarse al proyecto del girondino Condorcet, que pretendía la

(10) PUELLES BENÍTEZ, M., Op. Cit., pp. 66-69.

(11) Apoyaremos parte de nuestras aseveraciones en sendos estudios que dos profesores de la Universidad de Cádiz han publicado en relación a la historia de la educación en esta capital, me refiero al libro de ROMÁN, R. La educación en Cádiz en el siglo XVIII, Cádiz, Unicaja, 1991: y la Tesis Doctoral del profesor JIMÉNEZ GÁMEZ, R., La Sociedad Económica gaditana y la educación en el siglo XIX. Caja de Ahorros de Jerez, 1992.

(12) ROMÁN, R., lo ha tratado convenientemente, extrayéndolo de las sesiones municipales celebradas el 1 de julio y el 22 de septiembre de 1809, Op. Cit., pp. 48-51. 
existencia de centros de preparación específicos para el magisterio, de los que nacerían las futuras escuelas normales, las cuales, a partir de la ley de instrucción de 1857, trasplantaremos directamente a nuestro país, después del éxito de la experiencia piloto madrileña de 1838 .

Tambiên hay que añadir que el informe de los gaditanos incluye un apartado especial que se refiere a la educación de la mujer, a la que se le reconoce el derecho a una instrucción elemental que no sólo abarque las labores "propias de su sexo", en expresión de la época, sino que atienda a la lectura, escritura, aritmética y religión. Se expone que los conventos de monjas serían los lugares idóneos para impartirla y se fundamenta todos estos postulados con el criterio ya conocido de que no se trata de una instrucción para sí, sino que esta educación la encaminaría a afrontar con éxito la formación de un tercero, el hijo, futuro súbdito o ciudadano $y$, en todo caso, contribuiría a capacitarla para todos los asuntos que atañen a la economía doméstica de sus hogares (Vid. nota 5).

\section{LA REALIDAD ESCOLAR}

Desde que en 1566 tanto el cabildo catedralicio y como el municipal acordaran sufragar los gastos que ocasionara la instalación de una escuela auspiciada por la recién creada orden jesuítica, la enseñanza de los gaditanos en sus primeras fases quedaba confiada a la labor que dichos Padres desarrollaran en la Orden, que en el XVIII ya contaba con unas ocho centenas de jóvenes recibiendo instrución dentro de sus muros (13). En este siglo, los privilegios otorgados con regia sanción al gremio de maestros de San Casiano (al menos caben citar los ordenamientos establecidos en 1713, 1754 y 1773), preservaban el derecho de abrir escuela a determinados individuos, prohibiendo la libre competencia y vigilando estrechamente a quien, sin el debido permiso, pretendiera dedicarse a la enseñanza, acaparando para sí una parte del mercado de alumnos al que no se tenía derecho.

Prácticamente durante todo el siglo, nos dirá Rafael Román, funcionarán con el debido permiso de la autoridad y de la Hermandad 9 escuelas particulares de varones sin que exista ninguna variación en su número. Las novedades, en cuanto al régimen de los centros existentes en el XVIII vendrán dadas por el decreto de expulsión de la Orden de San Ignacio de Loyola firmada por Carlos III en 1767 , entonces, el interés por dirigir la antigua escuela de los jesuitas llevará primero a que el gobierno acepte la propuesta de un particular, José Carbonell y, más tarde, la de Francisco Antonio Domínguez que la supervisarán hasta 1785, fecha en la que se deja paso al obispado que es el que oferta unas mejores condiciones para hacerse cargo de ella. Bajo jurisdicción episcopal estará hasta que, con el estallido del conflicto hispano-francés, pasaría a convertirse tristemente en cuartel-vivienda de la tropa residente en la ciudad.

(13) Sobre la trayectoria educativa de la Compañia de Jesús en esta ciudad, vid., AZCÁRATE RISTORI, I., "Historia escolar de Cádiz 1564-1767 o raíces de un pueblo" en Tavira, Revista de la Escuela Universitaria de del Profesorado de E.G.B., "Josefina Pascual", Cádiz, 1993, № 10, pp. 79-94. 
Años antes, hacia 1792, la iniciativa de algunos particulares de cierta relevancia social en la ciudad, preocupados por el estado de la instrucción de la juventud gaditana, como el regidor D. Francisco Huarte, habían conseguido traer a la ciudad a D. Juan Rubio, director de las Escuelas Reales de Madrid con el objeto de implantar en la ciudad el mismo método que se empleaba en los centros de enseñanza de la Corte (14). A raíz de esto, y coincidiendo con el cambio de siglo, unas nuevas ordenanzas en forma de Estatutos del llamado Colegio Académico de Primeras Letras intenta, con aprobación real, reglamentar las condiciones de la enseñanza primaria en la ciudad (15). El Reglamento, en el que sería muy largo entrar, concedía a sus socios de número fundamentalmente, directores y maestros de las escuelas de la ciudad, el control sobre todo lo concerniente al magisterio, funcionando verdaderamente como una asociación gremial preocupada sobre todo por vigilar el número de licencias expedidas para ejercer la profesión y en pugna, ciertamente, con el centro Pío, la antigua escuela de los jesuitas, todavía bajo jurisdicción del cabildo catedralicio, cuyos maestros, apoyados por el Obispo, se negarán a cumplir las exigencias que el Colegio Académico se cree en la obligación de imponer, tales como la asistencia las tardes de los jueves a unas reuniones orientadas hacia la formación de los maestros, ayudantes y pasantes de todas las escuelas de la ciudad (16).

(14) ROMÁN, R., Op. Cit., p.91.

(15) Por Provisión Real de 22 de diciembre de 1780 había sido suprimida la antigua hermandad gremial de San Casiano y sustituida en la Capital del Reino por el corporativismo de un nuevo Colegio Académico, encargado de examinar a los futuros aspirantes al magisterio público. La iniciativa gaditana que aquí vemos, como ocurrió con el intento de implantación del sistema de enseñanza seguido en las Escuelas Reales que funcionaban en Madrid, nos habla elocuentemente de la rápida puesta al día de la capital andaluza con respecto a las novedades de organización educativa emprendidas desde la Corte. Hemos extraído esta información de la Real Orden de 4 de julio de 1806, reproducida en las Actas Capitulares del Ayuntamiento de Cádiz, en adelante A.C.A.C., consultadas en el Archivo Histórico Municipal de Cádiz, 7 de agosto de 1806, p.5, ff. 420-423v.

(16) Reglamento del Colegio Académico de Primeras Letras de la ciudad de Cádiz, Imprímase Cueto, Cádiz, 2 de enero de 1802. Los Estatutos habían sido presentados para su aprobación el 18 de marzo de 1800 y el Real Despacbo del Supremo Consejo de Castilla que lo autorizaba tiene fecha de 11 de febrero de 1801, habiendo sido presentado en el cabildo de 7 de febrero, punto 8, por los Diputados de Escuelas D: Francisco Huarte y Conde de Casas Roxas, A.C.A.C., 1801, ff 143 18 - 180 .

El conflicto entre los representantes del antiguo gremio de. San Casiano y la autoridad catedralicia puede verse en el Expediente por acuerdo del Ayuntamiento sobre oficiar al Ilmo. Sr. Obispo de esta Diócesis a fin de que concurran a los actos académicos del Colegio de Primeras Letras los ayudantes de las Escuelas Reales y demäs que con respecto a su Dirección en el mismo se trata, C.4770, reúne un 'conjunto de comunicaciones cruzadas entre las diversas autoridades implicadas desde finales de 1801 a comienzos 1802. El Ayuntamiento, por su parte, parecía también formar parte del frente abierto contra el Obispado cuando en el pleno de 14 de diciembre, punto último, había encomendado a una comisión, encargada de arbitrar la forma en que se debía encauzar la instalación de tres escuelas más ordenadas por el Supremo Consejo, el hacer partícipe al Obispo el descontento que se experimentaba por "el descuido en que se hallan las Escuelas Reales que fueron de la dirección y gobierno de la extinguida Compañía con el nombre de Jesús", A.C.A.C., 1801, ff. 681-681v. 
Sin embargo, pocos años más tarde, los días del Colegio Académico de Primeras Letras estarán contados y la Monarquía, deseosa de acabar con las limitaciones a la libre competencia que imponían este tipo de asociaciones corporativas decretará su disolución definitiva en el mes de julio de 1806, argumentando explícitamente los efectos nocivos que procuran y la estrechez de miras con que dichos colegios se conducen, siendo sustituidos por las llamadas Juntas Provinciales de Escuela, presididas por el Gobernador e integradas por dos prestigiosos maestros en cada capital provincial, que tendrian a partir de ese momento la potestad de examinar a los futuros aspirantes al magisterio y, encontrándolos aptos, elevar sus pretensiones al Consejo de Castilla que, después de comprobar las certificaciones exigidas de limpieza de sangre, buenas costumbres, moral intachable y exacto conocimiento de la doctrina cristiana, expedirían el título correspondiente habilitándoles para abrir escuela allá donde tuvieran por conveniente, sin que institución o cuerpo alguno pudiera impedírselo (17).

Las resistencias de los colegiales y las protestas del Ayuntamiento a la orden real fueron muchas, puesto que es evidente que éste último se servía del Colegio para fijar las condiciones educativas dentro de la ciudad. Son constantes las quejas de nuestros regidores acerca de la ruina que la indiscriminada apertura del magisterio podía traer tanto en el descenso del nivel de la instrucción, ya que se ignoraba a qué método se acogería el recién-llegado, como en el descalabro que podian sufrir los recursos medios de cada profesor al tener que compartir la misma clientela fija de la ciudad con la cohorte de nuevos recién llegados que se esperaba. La pérdida del control en la designación de maestros llevará al Ayuntamiento, una y otra vez, a solicitar de los monarcas, por un lado, el utópico restablecimiento el Colegio Académico con su régimen estatutario aprobado y, por otro, más conciliadores con la realidad, la presencia de dos regidores municipales como vocales de la Junta Provincial de Examen, entendiendo que sujeta a la voluntariedad y arbitrariedad de los dos maestros que son sus vocales la designación de los candidatos, ésta podría estar mediatizada por envidias o gustos personales (18). Las peticiones, reiteradas una y otra vez con machacona insistencia, serán desestimadas por la autoridad central en cada ocasión (19). Es obvio que

(17) Como se decía: "siendo cada maestro dueño de establecer su escuela en el cuartel, barrio, calle o lugar que bien le pareciese, sin que los maestros de número puedan oponerse a ello a pretexto de sus privilegios o estatutos", el objetivo perseguido por esta Real Orden rubricada en Cádiz por el Marqués del Socorro en agosto de 1806, era el de "evitar todo espíritu de cuerpo", Actas de la Junta de Estudios y Escuelas, sesión de 6 de septiembre de 1806, ff. 39-49. C.4770. A.H.M.C.

(18) El Informe evacuado por la Junta de Escuelas, una vez conocido el Decreto de disolución, se expresa en estos términos y muestra sus reticencias ante la conveniencia de la disposición regia, A.C.A.C., 22 de septiembre, de 1806, p.3, ff. 501-521. Finalmente, la aceptación del Ayuntamiento será cursada cuando se haga firme la orden del Gobernador, Ibidem, 25 de octubre de 1806, p.17, ff. 602-607.

(19) Todavía aparecen quejas en el Dictamen presentado por la Comisión municipal formada por el Regidor perpetuo y el Diputado del Común para su aprobación y elevación al Supremo Consejo, A.C.A.C., 21 de enero de 1815, p.8, ff. 44-45v.

A mediados de 1815, la Comisión de Escuelas, encargada de elaborar el informe final sobre la aprobación de la Escuela Gratuita que el Ayuntamiento solicita del Supremo Consejo, 
con ello, se había dado un paso decisivo para crear las condiciones de libertad que más adelante serán estipuladas por el discurso doceañista.

En cuanto al número de centros de instrucción en el preludio del nuevo siglo, sabemos por el Reglamento del Colegio Académico que éste permitía la existencia de 17 establecimientos masculinos en total, de los que 14 eran escuelas de primeras letras regentadas por maestros particulares, dos eran Academias de idiomas'y, hasta que la crisis bélica cerrara el centro de la Compañía de Jesús a cargo del Obispado, éste completaba el número de las existentes. Los redactores del Reglamento consideraban, de todas formas, insuficiente la oferta educativa, reconociendo la conveniencia de erigir escuelas en las zonas más estratégicas, por depauperadas, de la ciudad: barrio de Santa Maria (plazuela de la Merced), Barrio de la Viña (calle de la Palma), Barrio de San Carlos (plazuela del pozo de las nieves) (20). Sin embargo, pese a estas reflexiones y a las expectativas creadas por la labor normativa de las Cortes constituyentes, parcas fueron las realizaciones de nuestros ediles que se mostraron poco consecuentes con el programa educativo elaborado en 1809. La escuela Pía ocupada por las fuerzas defensivas permanecieron sirviendo de cuartel por bastante tiempo. Tan sólo, hacia 1813, el Ayuntamiento retomará el proyecto de abrir el centro que requerirá, como podemos suponer, obras de reconstrucción para alojar de nuevo a los numerosos escolares que se esperaba llenasen las aulas que se abren con la intención de recoger a las clases menos favorecidas de la ciudad, a la que se garantizará la gratuidad de la enseñanza (21). Es evidente que se pensaba recuperar la clientela de desheredados que acudían a la vieja escuela de los jesuitas. En los tiempos en que las autoridades del Colegio Académico y el Obispado estaban en pugna por la observancia o no de unos estatutos particulares, como hemos visto, el Obispo Escalzo, había salido al paso de las acusaciones vertidas en contra del magisterio que destinaba para aquella obra, advirtiendo que a ésta sólo acudían "un enjambre de muchachos pobrísimos, enfermísimos, descalzos y desnudos, de mala crianza, 'que no pueden costear los libros, papel, plumas, tinta y lo demás necesa-

introduce opiniones particulares sobre posibles mejoras en el ámbito educativo que pasan, entre otras actuaciones, por el restablecimiento del viejo Colegio, A.C.A.C., 8 de abril de 1815, p.14, f. $315 \mathrm{v}-333$.

En el mismo sentido, en escrito del Sindico Personero remitido al Gobernador de la Plaza, en su calidad de Presidente de la Junta de Escuelas, tras describir el desorden que atañe a la primera educación de la ciudad, sometida a la arbitrariedad de los maestros de la misma, solicita que dos regidores, más el Síndico Personero formen parte de la Junta, como elementos imparciales en los tribunales de aprobación de los candidatos, al mismo tiempo que es presentado los Estatutos del Colegio Académico, cuyo restablecimiento se interpone ante tanta parcialidad que se describe. Ibidem, 26 de mayo de 1815, ff. 395-396.

(20) Reglamento... Op. Cit., p.30.

(21) La creación de la nueva Escuela Gratuita "para jóvenes indigentes", que debía ocupar, por tanto, el edificio jesuítico, había sido encomendada a una Comisión municipal que había ido informando puntualmente de los trabajos realizados en diferentes plenos celebrados a lo largo de ese año (Vid. $\mathrm{N}^{\mathrm{N}} 39,50$ y 51) hasta conseguir la aprobación de su Reglamento el 12 de junio, punto 16, A.C.A.C. 1813 , ff. $124 \mathrm{v}$. En días sucesivos se saca a concurso la plaza de director a la que se presentan 8 candidatos (16 de junio, punto 2, f. 128) y son nombrados 6 ayudantes, a los que se les expide título correspondiente (11 de agosto, punto 6, f. 179v). 
rio y que sólo asisten alguno que otro día que sus padres no los necesitan para buscar su vida infelizmente, o que ellos no pueden trampear su asistencia a la escuela como lo hacen con frecuencia" (22).

Sobre la escuela, abierta desde primeros de septiembre de ese año con la asistencia de "más de 200 niños" el día de su inauguración, que , al poco, superaban los 800 , pendió desde el principio la amenaza permanente de cierre, y es que el Ayuntamiento se las vio y deseó para poder financiar sin gravar la partida de propios aquel centro escolar (23). Se impuso un impuesto sobre las entradas a los teatros gaditanos, pero pronto esta fuente de ingresos se mostró insuficiente (24). Se llegó a desviar đerechos que se cobraban en las fuentes y aljibes (concretamente el de la plaza de San Fernando), por último, de los santanderinos, regentes de las tiendas de ultramarinos, más conocidos por "montañeses" se requirieron los recursos necesarios. Todo, sin embargo, parecía insuficiente y una vez restablecida la monarquía fernandina, el cambio en las autoridades locales subsiguiente que se produjo, deja entrever cómo la escuela se considera necesaria pero un

(22) Carta del Obispo Escalzo dirigida al Ayuntamiento en respuesta a las acusaciones de los maestros del Colegio Académico, que, según se llegaba a decir sólo reciben "un corto número de niños muy decentes, hijos de Padres Poderosos que asisten todos los días y pueden costear cuanto necesiten para su enseñanza a que se agrega la mejor índole que tienen para recibirla, por su cuna, su educación y su clase". La misiva está tiene fecha de 11 de enero de 1802 y se encuentra en la C. 4770 , del A.H.M.C.

(23) El 1 de septiembre de 1813, se procedió a su apertura, aceptando a "todo joven mayor de seis años que demuestre su indigencia con papeleta del Comisario de Barrio" y solicitando de los padres "el celo necesario para que los niños acudan", Ibidem, 14 de agosto de 1813, punto $14, \mathrm{f} .184 \mathrm{v}$.

Las cifras de la matrícula están extraídas del Informe del comisionado por el Ayuntamiento D. Joaquín Antonio Gutiérrez de la Huerta, procurador mayor, para evaluar las alternativas de financiación de la escuela gratuita que se intentan sean aprobadas por el Supremo Consejo, Ibidem, 8 de abril de 1815, ff. 319-333. En el mismo, poniendo de manifiesto que el cambio institucional del Cabildo efectuado el 30 de julio de 1814 no había modificado el interés de la nueva Corporación pơr esta escuela, pero encontrando exhausto el caudal de propios de que se nutría, proponía el gravamen sobre las entradas a los teatros cómicos de la ciudad, razonando que, al ser frecuentados éstos por personas pudientes, éstas contribuían con justicia al mantenimiento de una escuela para pobres, calculando en 50.000 reales de vellón las ganancias que podían obtenerse al año por esta vía. El Informe tiene fecha de 30 de diciembre de 1814.

(24) La Comisión de Escuela informaba puntualmente de los gastos ocasionados mensualmente por el centro escolar entre sueldos y material. Se vivía pendiente de la recaudación del teatro del Balón, de sus funciones de volatines, operas, sombras chinescas y hasta bailes para satisfacer entre tres mil y cinco mil reales que, mes a mes, consumía la escuela. Ibidem, 1 , 22 y 29 de septiembre de 1813, p.8 (f. $198 \mathrm{v}$ ), 3 (f. $216 \mathrm{v}$ ) y 6 (f. 224), respectivamente.

\begin{tabular}{|c|c|c|c|c|}
\hline Octubre ......... & $3.674 \mathrm{rs}$ & 30 , oct., & p.7, & f. 247 \\
\hline Noviembre & 3.377 rs., & 1, dic., & p.6, & f. $273 \mathrm{v}$. \\
\hline Diciembre ................ & 4.579 гs., & 5 , ene., & p. 16 & f. 6 \\
\hline Enero & 3.584 rs., & 3, feb., & p.14, & f. 47 \\
\hline Febrero & 3.760 rs., & 2, mar., & p.6, & f. $78 \mathrm{~V}$ \\
\hline Abril .... & 5.324 rs., & 4, may. & p. 10 , & f. $145 \mathrm{v}$ \\
\hline Mayo & 3.317 rs., & 15, jun., & p.21, & f. $176 \mathrm{v}$ \\
\hline Junio & 4.563 rs., & 16, jul., & p.17, & f. 200 . \\
\hline
\end{tabular}


verdadero lastre para el erario municipal (25). De manera que, desde entonces, el nuevo Cabildo se marque el objetivo de deshacerse de ésta, recabando las garantías suficientes para que el establecimiento permanezca abierto.

Se pensará en primer lugar en la recién formada Sociedad Económica de Amigos del País, que desde sus comienzos, como ha estudiado el profesor Rafael Jiménez Gámez, nace con pretensiones educativas muy a tener en cuenta. Volcada hacia proyectos novadores, en cuanto a los sistemas pedagógicos que va a aplicar en sus escuelas, concretamente habrá que mencionar la puesta a punto en sus aulas del método mutio o lancasteriano que tanta importancia va a tener para la historia didáctica de lo que queda de siglo, se hará cargo del centro en los meses de 1818 (26). Al mismo tiempo que se mantienen conversaciones con la Sociedad, el municipio solicita al soberano el restablecimiento de los jesuitas, puesto que Fernando VII había levantado la prohibición que pesaba sobre la Orden. Confiaban, nuestro ediles, que dicha petición podía hacer olvidar a la monarquía el papel que la ciudad había protagonizado en la España Liberal en guerra y, al mismo tiempo, si se conseguía que los Padres se hicieran cargo con sus propios recursos del antiguo centro que dirigían en la ciudad, se dispensaría de un importante gasto a las siempre parcas arcas municipales (27). En la solici-

También en el A.H.M.C., C.4823 se conserva el legajo que especifica las cuentas de la escuela gratuita desde 1814 hasta 1819, haciendo públicas las fuentes de financiación: venta de agua, impuesto a los montañeses, impuesto a las diversiones públicas, como teatros y corridas de toros, incluso, multas a los teatros y a los actores. En 1814, el gasto había ascendido a 65.333 reales de vellón, en 1816 se gastaron 72.947 y en 1818 la cantidad se redujo considerablemente a 43.633 , gracias, presumiblemente, al concurso de la Sociedad Económica que termina haciéndose cargo del centro.

Por su parte, el director de la misma evaluaba con absoluta escrupulosidad el gasto mensual de la escuela que se desglosaba de la siguiente manera:

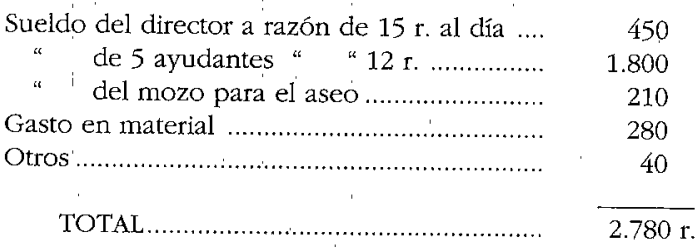

Estas cifras,tomadas de un mes correspondiente a 1817, se repetirán sin apenas variación entre 1814 y 1818, años para los que también existen datos mensuales. A veces, se alcanza los 3.000 rs., incluso hay meses de 4.000 y la razón siempre se encuentra en algún gasto extraórdinario, normalmente, en el capítulo de compra de material y libros.

(25) La Diputación de Escuelas no tardaría en denunciar la falta de fondos con qué pagar los gastos ocasionados, a lo que el Ayuntamiento contestaría que, falto de recursos, sólo cabía esperar la celebración en la ciudad de otra función que diera dinero, Ibidem, 1 de febrero de 1815, p.5, f. $97 \mathrm{v}$ y 98

(26) JIMÉNEZ GÁMEZ, R., Op. Cit., pp. 142-151.

(27) El Expuesto presentado, en este sentido, por el procurador mayor D. Joaquín Antonio Gutiérrez de la Huerta, entre otros firmantes pertenecientes a la Corporación, al Cabildo celebrado el 24 de octubre de 1814 , p. 22 , A.C.A.C. f. $425-430 \mathrm{v}$, fue aprobado y elevado a Su Majestad sin demora 
tud cursada se podía leer, tras hacer un canto laudatorio de la actividad docente desplegada por "ese cuerpo Religioso de varones apostólicos y de maestros universales", que sólo la desorganización de la red escolar se había conseguido con su marcha forzada, al mismo tiempo que el "republicanismo" y la funesta influencia traídas por los "novadores", operaban en contra de los principios monárquicos y religiosos que sustentaban el orden social. La vuelta, unilateralmente solicitada por el Ayuntamiento, pretendía cumplir sobradamente con los objetivos anteriormente explicitados, congraciarse con el Rey restituido desagraviándole de las actuaciones del liberalismo gaditano, reorganizar una red escolar para la ciudad con el menor coste para el Municipio y, finalmente, pero no menos importante, garantizar la inculcación en el espacio escolar de la triada "altar, patria y trono", en un intento de neutralizar las peligrosas teorías desestabilizadoras que habían conmovido a la Europa de los últimos tiempos.

Efectivamente, a comienzos de 1819 , los jesuitas, ya de regreso e instalados en sus antiguas pertenencias: la Iglesia de Santiago y el Seminario de San Bartolomé, abrían su escuela a 200 niños que al poco pasarían a ser 800 . La presión de esta demanda hará que los Padres de la Orden soliciten, sin éxito, recursos al municipio por la imposibilidad de garantizar la gratuidad para tantos niños. Concretamente el auxiliar de la Conpañía daba a conocer al Ayuntamiento que no había medio de mantener la matrícula de 746 niños, y más que era de esperar se matriculasen, en el espacio que ocupaban. Atendida por 11 jesuitas, 6 seglares con paga diaria, un maestro, 4 ayudantes y un capellán, se iban a sumar más jesuitas venidos de Italia, por lo que se reclamaba ayuda urgente al Municipio, ayuda que nunca satisfizo (28).

Por lo que respecta a la enseñanza, si no privada, sí ejercida por particulares, el recuento total de escuelas abiertas hacia 1813 daba 31 centros de enseñanza primaria masculina, 90 del tipo de "amigas" y academias femeninas y 12 establecimientos de enseñanzas de gramática, latinidad y lenguas (Vid. cuadro № 1 al final). Hacia 1818, las escuelas elementales apenas habían variado: 35 para los niños, 100 para las niñas y párvulos recogidos en las "amigas". Por su parte, los maestros de latinidad, gramática y lenguas eran contabilizados con detenimiento hacia 1815, siendo objeto de la siguiente clasificación: estudios de latinidad propiamente dicho, es decir los únicos con derecho a la existencia (7), Academias que enseñan latinidad sin tener a sus preceptores y directores examinados de latindad, bellas letras e idiomas (5), establecimientos de primera educación donde se enseña latinidad clandestinamente (4) y enseñantes de latinidad privados sin titulación (10) (29).

Una vez solventadas las restricciones corporativas, si tratamos de averiguar, a continuación, cuantos niños y niñas del conjunto de la población infantil gaditana por aquellos años acudían a la escuela, es decir, si pasamos a estimar el nivel de escolarización alcanzado entre todos los establecimientos que anteriormente hemos mencionado, nos topamos con la dificultad de no encontrar suficiente información al respecto, debido seguramente a lo aleatorio de la matrícula y de la asistencia de

(28) A.C.A.C., 15 de junio de 1819, p.3, ff. 536-537.

(29) A.C.A.C., 15 de julio de 1815, p.19. Se contesta a la solicitud expresada en el Cabildo de 30 de junio sobre maestros de latinidad existentes en la ciudad, ff. 584-587. 
los escolares, que normalmente se retiraban cuando sus conocimientos comprendían la lectura y la escritura $o$, incluso, antes de dominar dichas destrezas si el desempeño de un oficio, actividad mucho más rentable a corto plazo que éstas, salía al encuentro. Tenemos datos para comienzos de siglo y para 1818 que nos pueden arrojar cierta luz sobre este punto. La presencia de las niñas y niños en las casas de las "amigas", academias de señoritas, Casa de la Misericordia, escuela del Callejón de la Cerería que fundara en 1787 Doña $M^{a}$ de Arteaga podría llegar a ser de 1.940 escolares en 1801. Por su parte, los varones que asisten a las escuelas de primeras letras, al colegio de la Compañía y a la misma Casa de la Misericordia, son estimados en 1.757 (30). Un informe completo de la situación escolar gaditana elaborado por una comisión municipal en 1818 daba estas cifras: los centros femeninos que se circunscribían a los ya citados anteriormente apenas sí habían variado su matrícula, siendo frecuentados por 2.079 niñas. Los niños; en cambio, con 35 locales particulares abiertos, incluido el centro gratuito que en la calle de la Rosa mantenía la Sociedad Económica de Amigos del País desde julio de 1818, la Casa de la Misericordia y la escuela gratuita municipal, provisionalmente también gestionada por la Sociedad, crecen singularmente hasta 2.809 matriculados (31).

La tasa de escolarización, es decir, la razón existente entre alumnos matriculados y población infantil susceptible de escolarizar, depende de las edades límites que escojamos como apropiadas. Normalmente, para estas fechas establecemos un segmento entre los 6 a los 12 años, en el que podía muy bien un niño o una niña pasar en algún centro escolar un período indeterminado. Con los padrones y recuentos vecinales a la vista es posible evaluar el número de ese grupo de edad:

$\begin{array}{lll} & \text { NIÑOS } & \text { NIÑAS } \\ 1801 \ldots \ldots \ldots \ldots & 3.684 \ldots \ldots \ldots \ldots \ldots . & 3.704 \\ 1813 \ldots \ldots \ldots \ldots & 4.187 \ldots \ldots \ldots \ldots \ldots . & 3.848\end{array}$

Fuente: cifras censales para 1801 estimadas por Rafael Román, Op. Cit., pp. 58-59; población censal de 1813 estipuladas a partir de los datos ofrecidos por Julio Pérez Serrano, Cádiz, la ciudad desnuda. Cambio económico y modelo demográfico de la Andalucía contemporánea, Servicio de Publicaciones de la Universidad de Cádiz, 1992, pp. 224 y 226.

Haciendo la oportuna relación entre las dos magnitudes que tenemos, población escolar y matrícula, Román concluye que el $47 \%$ de los niños entre 6-12 años asistían a clase en 1801 , porcentaje que se verá incrementado hasta un $67 \%$ en 1818; según nuestra propia estimación para ese año. Las niñas, en cambio, apenas cambian sus actitudes: un 52\% en la primera fecha, un $54 \%$ en 1818 porcentaje que seguramente es superior al real puesto que no tenemos medios de descontar a los párvulos de ambos sexos, menores de 6 años, que acudian diariamente a las "amigas".

(30) Las cifras son facilitadas por Rafael ROMÁN, Op. Citt., p.59. Las cantidades de Ramón SOLÍs que se sirvió de la Relación de gremios y número de individuos de 1801 ofrece la misma descompensación en favor de las niñas que son 1.828 , mientras que los niños alcanzan las 1.594 matrículas, SOLÍs, R., El Cãdiz de las Cortes, Silex, 1987, p.311.

(31) JIMÉNEZ GÁMEZ, R., Op. Cit., p.136. 
No debemos ser demasiado optimistas, a la hora de interpretar estos porcentajes. Por un lado, El profesor Román nos habla de posibles ocultaciones en el padrón que harían descender la tasa entre 5 y 10 puntos, por otro, la escolarización femenina merece un comentario particular sobre la naturaleza de los centros que las acogen. Estos establecimientos responden esencialmente a dos modalidades, las llamadas escuelas de "amigas" y las "Academias de señoritas", son centros donde no se ofrece más que una educación de "adorno", caso de las academias, y sobre todo una extensa formación y exclusivo aprendizaje en las labores de costura y bordado, en el recinto de la "amiga" o "miga", como vulgarmente son denominadas, de manera que lo que conocemos como educación literaria, la enseñanza de la lectura y la escritura brillarían por su ausencia, en vista de lo cual habría que tomar con mucha precaución las posibilidades de alfabetización efectiva de esta población escolar femenina (32).

La precariedad de lo exigido a las futuras candidatas a regentar una "amiga" así nos lo demuestra. Se trata normalmente de una mujer de la que no queda garantizado sus conocimientos literarios y que tan sólo es examinada de labores por las maestras que atienden la Casa de la Misericordia de la ciudad. Previamente han presentado un certificado del cura párroco que las habilita en sus conocimientos de doctrina cristiana y lo propio del comisario del barrio que da fe de su buena moral y sanas costumbres. Aparte de esto, la petición hecha al ayuntamiento nos descubre invariablemente que la solicitud la promueve en última instancia un estado particular de desesperación y precariedad económica: bien son viudas, huérfanas, con padres o hijos a su cargo, abandonadas, etc... lo que hace que normalmente al ayuntamiento le sea muy difícil negar la licencia solicitada. Su número llegará a sobrepasar la centena y de ello se apercibirá el Colegio Académico que, temiendo por la propia continuidad de las existentes, aconsejará la desestimación de nuevas peticiones recomendando una reducción considerable de su número a fin de que pueda subsistir sus maestras de forma decorosa (33).

La esperada y reiterada reforma en este capítulo se hará esperar por bastante tiempo y únicamente la competencia de centros escolares más modernos y reglados, preparados para dar una formación a la mujer algo más completa hará descender, de forma paulatina, el número de amigas abiertas en la población, de modo que a mediados de siglo podrían llegar a ser una treintena larga. Pensamos que debieron funcionar como verdaderos parvularios o guarderías para niños y niñas menores de cuatro años que son confiados por aquellas mujeres que trabajarian fuera del hogar a las conocidas, "amigas", del barrio que se dedican a este menester. Teniendo estipulado no admitir a niñas mayores de 12 años, estamos por asegurar que las "amigas" no enseñaban a comienzos de siglo a leer y a escribir, por cuanto que en las licencias concedidas por la autoridad competente siempre se pide que la amiga designe a un maestro de la ciudad para que se encargue

(32) Ofrecemos una descripción de estos centros en ESPIGADO TOCINO,G., "La población escolar femenina a mediados del siglo XIX en Cádiz" en VII Encuentro de la Ilustración al Romanticismo. La mujer en los siglos XVIII y XIX, Servicio de Publicaciones de la Universidad de Cádiz, 1994, pp. 201-212.

(33) Reglamento... Op. Cit., p. 4. 
de dicha instrucción en su escuela. La continua designación de los mismos nombres de pasantes para un sin fin de casos que hemos verificado cuestiona que este tipo de instrucción se contemplase efectivamente.

Llegados a este punto, poco hemos visto, en cambio, acerca de cómo era en realidad la vida en el interior de las aulas, bajo qué régimen, con qué libros, qué materias, con qué horarios.. se sometían, como hemos visto, al menos a la mitad de los gaditanos y gaditanas en edad escolar a la disciplina del aprendizaje. De modo breve, el Reglamento elaborado por la Diputación de la Escuela Gratuita municipal para funcionamiento del centro que dicho organismo abre en septiembre de'1813, nos puede servir para responder a alguna de estas cuestiones (34).

Los varones que acudian a este establecimiento tenían clase durante todo el año, pues no se conocía el receso correspondiente a la vacación estival, de $81 / 2$ a 11 de la mañana y de 3 a 6 de la tarde, entre abril y septiembre y de 9 a 12 de la mañana y de 3 a 5 por la tarde, entre octubre y marzo. Puesto que la enseñanza era absolutamente gratuita, así como el material necesario (libros, papel, plumas y tinteros eran repartidos por el Director), era imprescindible para ser admitido en ella la acreditación como pobre de solemnidad expedida por los Comisarios de barrio. De hecho, en el Art. 11 se prohibía expresamente que los maestros cobrasen cantidad alguna a los padres, abominando del trato discriminatorio que solía acarrear la distinción entre niños de pago y gratuitos.

La dirección del establecimiento estaba en manos de un maestro principal cuya dotación diaria alanzaba los 25 reales. Bajo su jurisdicción, un ayudante, maestro segundo, que ejercería en su ausencia la labor de control y cobraba a razón de $15 \mathrm{r}$. por jornada, frente a los 12 que percibía el resto de los ayudantes. Un mozo encargado de la limpieza, aseo y suministro del agua, que cobraría a razón de 7 r. diarios, formaba parte, también, del personal destinado a su servicio. un dato a tener en cuenta era que, mientras la plaza de director era cubierta por el procedimiento que arbitrara el Ayuntamiento, la de los ayudantes se sujetaban al examen u oposición correspondiente.

Los niños llegaban a las aulas, al menos, los más pequeños, recogidos en sus casas y conducidos en filas de a dos por los ayudantes del colegio y, de nuevo, a la salida, eran repartidos por ellos. Se intentaba evitar la algarabía infantil a toda costa, obligándoles al saludo ante todo "sujeto de carácter" que saliese a su paso. otra medida disciplinaria prohibía a los niños salir de clase para efectuar sus "diligencias corporales", en evitación de la "indecencia" del acto, así como de las molestias y perjuicios que para la salud pública ello podía causar.

Las clases, como digo, duraban todo el año (incluidos los jueves, día de asueto normal en épocas pasadas, y los días de misa) y solamente quedaban francos de asistir los Domingos y fiestas y, claro está, el día del patrón del antiguo gremio de maestros que no era otro que San Casiano.

Dentro de la distribución del tiempo escolar es lógico pensar que las prácticas religiosas ocupasen buena parte de éste. La asistencia a misa con sus profesores, al menos una vez a la. semana, era una costumbre que perdurará durante buena parte del siglo. La última media hora de la clase vespertina estaba dedica-

(34) El Reglamento de la Escuela Gratuita, se encuentra inserto en la sesión municipal de 3 de octubre de 1814, ff. 322-323v. 
da el rezo del rosario y los sábados se empleaba exclusivamente en la enseñanza de la doctrina cristiana.

Si nos imaginamos que el sistema de enseñanza seguía los modos jesuíticos de instrucción, los niños estarían divididos en clases donde se les instruiría sucesivamente, primero a leer, mediante el deletreo y posteriormente a escribir. La aritmética se reduciría probablemente a las cuatro reglas, lo que en el Reglamento se especifica como contar y, como novedad, se señala que algunos rudimentos sobre gramática castellana serían introducidos. Era importante, según queda especificado, utilizar un mismo sistema para proporcionar estos conocimientos elementales. Por supuesto, la doctrina cristiana se encuentra incorporada desde el principio y su presencia es total si pensamos que los niños aprendían a leer sobre catecismos o libros de doctrina, e igualmente transcribían a sus planas máximas de moral religiosa. Cada 15 días el director estaba obligado a remitir un parte de altas y bajas, faltas de asistencia (el máximo disculpadas antes de ser borrados de la matrícula era de 8) a la Diputación de Escuela, indicando el pase de los alumnos de unas clases a otras.

\section{A MODO DE CONCLUSION}

Recapitulando en unas breves líneas, hemos presentado el modelo teórico que en los inicios de la revolución burguesa europea pretende abrirse paso también en nuestro país, modelo que se propone atender las necesidades de esa nueva sociedad de clases y no de estamentos en formación, también nos hemos referido a los proyectos que en nuestra ciudad se intentan llevar a cabo. Frente a estas aspiraciones de cambio, la realidad descrita nos habla claramente de los avances y retrocesos experimentados en la búsqueda de la consolidación de ese modelo de educación pública que termina desvaneciéndose cuando, a la vuelta de los jesuitas, solicitada por el ayuntamiento, éste decline en ellos su responsabilidad. Hacia 1815, respondiendo a una encuesta formulada por la Junta Provincial de Primera Educación, respecto a las características de la Escuela Gratuita, éste último reconocía haber contraído "las obligaciones de Padre de los jốvenes indigentes del Pueblo" a los que había proporcionado maestros examinados y aprobados para que les suministrasen la primera educación, "sin tener esta Corporación otra intención en la parte de la enseñanza que la que cualquier Padre tiene con respecto a sus hijos", resulta sorprendente que esta relación paternofilial se relaje, hasta que, nuevamente expulsada la Compañía en el Trienio, se asuma definitivamente la responsabilidad que los legisladores doceañistas le tenían reservada (35). De manera que los interludios liberales y neoabsolutistas del primer tercio de siglo, influirian decisivamente en la puesta en práctica de los ideales revolucionarios. A partir de esta última fecha, todo quedaba por hacer. La construcción de la red pública municipal evoluciona a medida que avanzamos hacia la mitad del XIX, incorporando las escuelas dirigidas por la Sociedad Económica, una masculina y otra femenina, a su jurisdicción y ampliando la oferta educativa con la apertura de cuatro centros para niños y tres para niñas, dos parvularios y un centro de adultos poco después de promulgada la Ley Moyano. En la actualidad, el estudio de estos centros escolares durante todo el siglo XIX constituye nuestro presente trabajo de investigación, que esperamos dar a conocer en fecha próxima y del que estás páginas significan un avance del mismo.

(35) A.C.A.C., 21 de abril de 1815 , ff. $248 \mathrm{v}-250$. 


\section{Santa Cruz}

Antonio Peñuela

Cruz Verde, 7

Bartolomé Gómez Bustamante

San Vicente, 26

José Perea

Santa Lucía, 109

Juan Vizcaíno

Aserrador, 142

Rafael Rivera Verino

Cuna, 157

José Peña (latinidad)

Cardoso, 201

Rosa Guillén

San Vicente, 18

Valentina de Vargas

Desamparados, 62

Angela Cuer

Santa Lucía, 104

Micaela Gómez

San Francisco Javier, 116

Dolores Pilar de Molina

Margarita Inestrosa

San Francisco Javier, 178

Josefa Arisa Mozo

Cerería, 188

Cardoso, 202

Nuestra Señora de las Angustias

Pedro Verdugo

Camino, 89

M. $\underline{a}$ del Carmen Freire

Camino, 89

Ana Calvete

San Francisco, 97

López Isabel

San Germán, 157

María Cayón

San Servando, 163

Mundo Nuevo

José Claudio Romero

Plaza del Balón

Josefa Baquero

Torre, 164

Antonia Sánchez

Solano, 205

Manuela Moreno

Solano, 223

Manuela de la Paz Vogarín

Sacramento, 244

Vicenta González

Sacramento, 267

Magdalena Muñoz

Rosa, 291

Gertrudis Moreno

Belén, 316

Isabel Beudaler

Santísima Trinidad, 273

La Viña

Pedro Mallada

Josefa Brau

Callejón de los Carros

Josefa de la $\mathrm{Paz}$

Santa Catalina, 24

María Mozo

San Leandro, 77

Josefa Moreno

Jesús, María y José, 149

Micaela García

San Félix, 169

De la Carreta, 197

Ave María

Manuel Risueño

José de Moya

Magdalena Viola

Plaza de las Viudas, 143

María del Real

Plaza del Hondillo, 179

Leonarda Martínez

Nueva, 49

Amoradores, 104

Isabel Benítez

Detrás del Pópulo, 293

De la Posadilla, 303 
San Felipe Neri

Antonio Lodolo

Miguel de Zaldúa

María Jaén

Josefa Obia

María Fernández

Isabel Guerrero

Manuela Zevallos

Antonia González

San Lorenzo

José Alvarez

José Agreda

Juana M. ${ }^{\text {a }}$ Bezelu

Josefa Moreno

Juana Quesada

Bárbara Leal

Juana Pico

Rita Rapalo

Luisa del Río

\section{Cruz de la Verdad}

Lamberto Prat

M. aㅡ Candelaria Silva

Josefa Vargas Machuca

Margarita Maraval

Francisca Pérez

\section{El Pilar}

Antonio Moliné

Rosario Curado

Juana Fernández

María Salado

Luisa Forrada

San Antonio y Bendición de Dios

Basilio Carsi

José Vianco

Diego Ronero

Ildefonso Ortega

Pedro Moreno

Rosalía de Córdoba

Gertrudis Mauricio

Micaela Reina

Josefa Magariño

Manuela Mantilla

M. ${ }^{a}$ de la O Bernal
Plaza de las Viudas, 104

Santa Inés, 25

Amargura, 21

Tres Hornos, 73

Plaza de las Viudas, 100

Zanja, 108

Zanja, 118

Zanja, 124

Rosa, 48

Herrón, 85

Capuchinos, 3

Capuchinos, 6

Rosa, 38

Nazareno, 183

Herrón, 81

San José, 104

Torre, 151

Oleo 26

Plaza de las Viudas, 126

Angel, 179

Santa Rosalía, 212

Oleo, 29

Molino 127

Ahumada, 12

Husillo, 155

Oca, 161

Fideo, 184

Constitución, 11

Constitución, 15

San José, 49

San José, 68

San José, 71

Murguía, 169

San José, 57

Fideo, 6

Molino, 66

Marzal, 103

Del Atmo. (sic), 38 
Nuestra Señora del Rosario

Pascual Castellanos

Rosario, 109

Juan Sánchez

Beaterio, 144

Santiago Arana (latinidad)

Beaterio, 139

Ana Garrido

San Francisco, 59

Ana Ruiz

Catalina Castelli

Carnecería del Rey, 195

Rita Fernández

Rosario, 104

Madres del Beaterio

Rosario, 82

Gertrudis Merino

Beaterio, 134

Verónica, 170

\section{Capuchinos}

José Mediavilla

Capuchinos, 75

Josefa Peña

Capuchinos, 97

M. ${ }^{a}$ Juana de la Paz

Portería, 120

María Montoro

Misericordia, 175

\section{Candelaria}

Juan López Castro

Candelaria, 186

Juan José Romo

Comedias, 32

M. Juana Boorquez

Cobos, 160

Francisca Rubio

Villalobos, 151

Antonia Jiménez

Santiago, 140

\section{Santa María y Merced}

Juan Losada

Yedra, 63

Antonia Olla

Merced, 4

Antonia Aguilar

Merced, 18

María Bernal

Santa María, 81

Rosalía Ramírez

Torno, 177

Juana Abad

Torno, 185

Agustina Franco

Torno, 199

Josefa Jiménez

Botica, 132

Manuela Rodríguez

Santa Elena, 206

Liberata Cortés

Santa Elena, 209

\section{San Roque y Boquete}

José Rodríguez

Sopranis, 116

María Cárdenas

Mirador, 20

Antonia Manzano

Santo Domingo, 46

Juana Mejías

Sopranis, 97

Josefa Guerra

Sopranis, 109

\section{La Cuna}

José Moliné

Angel, 71

Rita Muga de la Cerdá

Ancha, 62 (academia)

Paloma de la Calle

Sacramento, 175

María Navatro

Sacramento, 134 
Santiago

Isabel Ortega

San Juan, 82

Micaela Argüelles

Puerto Chico, 98

Ignacia Montes

Melos, 150

Agustina Flores

Empedrador, 185

Antonia Mérida

Empedrador, 206

Juana García

Empedrador, 206

Josefa Cantos

Empedrador, 206

Antonio Castellanos

Rosatio, 109

Santiago Pérez Arana

Beaterio, 139

Jaime Vis

San José, 97

Tomás Rerney

Fideo, 15

Manuel Sánchez

Teniente, 15

Francisco Beroteann

Torre, 59

Carlos M. Lambique

S. Fernando, 151

Bartolomé Meléndez

San Félix, 164

Manuel Regato

Consolación, 212

(Maestro de lenguas)

(Maestro de gramática)

(Maestro de lenguas)

Jorge Sinthon

Hospital de Mujeres, 164

Antonio del Castillo

Escuelas

José Piña

Cardoso, 201

(" " ")

(Maestro de gramática)

(Maestro de lenguas)

(" " ")

(Maestro de gramática)

(" " ")

(Maestro de lenguas)

(Maestro de gramática)

(Maestro de latinidad)

Fuente: Maestros de Primeras Letras con clase abierta (1813) C. 4770 . A.H.M.C. 